\title{
Oxidant-antioxidant balance in asthma and the potential of antioxidant therapies
}

\author{
A. Di Stefano, I. Gnemmi, C. Vicari, B. Balbi
}

Monaldi Arch Chest Dis 2010; 73: 3, 96-98.

Divisione di Pneumologia and Laboratorio di Citoimmunopatologia dell'Apparato Cardio Respiratorio, Fondazione Salvatore Maugeri, IRCCS, Istituto Scientifico di Veruno, Veruno (NO), Italy.

Correspondence: Antonino Di Stefano, PhD, Fondazione S. Maugeri, IRCCS, Divisione di Pneumologia e Laboratorio di Citoimmunopatologia Apparato Cardio Respiratorio, Via per Revislate 13, 28010 Veruno (NO), Italy; e-mail: antonino.distefano@fsm.it

Numerous publications exist showing enhanced levels of oxidant production in asthma. Compared to control subjects $[1,2]$ asthmatic patients are characterized by increased levels of inflammatory cells that, in turn, produce more reactive oxygen species (ROS).

'Airway antigen challenge' is the experimental model that has been used in atopic subjects to study the mechanisms and mediators that result in asthmatic responses and airway inflammation [3]. Asthma attacks and experimental antigen challenge were found to be associated with immediate formation of ROS which persisted throughout the late asthmatic response [4]. Approximately ten minutes after the antigen was introduced into the airways, a two-fold increase in oxygen was observed. Spontaneous and experimental allergen-induced asthma attacks led to leukocyte (eosinophil, neutrophil) activation, during which NADPH oxidase was activated and $\mathrm{ROS}$ such as $\mathrm{O} 2 \bullet-$ and its dismutation product, $\mathrm{H}_{2} \mathrm{O}_{2}$, were rapidly formed. In addition, a link between the increase of ROS and asthma severity was evident. Indeed the severity of the hyperactivity was associated with the ROS production and increased neutrophil infiltration and activation [3].

A significant increase in neutrophils has been observed in the late-stage reaction following antigen challenge in a number of asthmatic phenotypes, e.g. fatal asthma, nocturnal asthma and long-standing asthma, and in those with steroid responsive intractable disease [5]. Modifications of MPO-mediated oxidant were also a contributing factor to the pathophysiology of severe asthma. A significant (2- to 3-fold) increase in chlorotyrosine was observed in subjects undergoing a segmental allergen challenge. Malondialdehyde and thiobarbituric acid reactive products were also identified in urine, plasma, sputum, and bronchoalveolar lavage fluid which, in turn, correlated with the severity of asthma. In addition, 8-isoprostane, a biomarker of lipid peroxidation, was also increased in exhaled breath condensate in both adults and children with asthma [5]. The rapid induction of protective antioxidant enzymes is an important factor as it determines the homeostasis of cellular functions during oxidative stress [6]. Cells and tissue are of course naturally protected from the constant production of reactive nitrosative species (RNS) and ROS by natural antioxidants which occur during metabolism. It is, however, important to note that the antioxidant defences may be overcome by increased levels of reactive oxygen species which, as a result, can cause oxidant-mediated injury and ultimately death of the cells. A number of studies have hinted at the important role that oxidative stress plays with regard to the initiation and progression of asthma. These studies have shown that variations to redox can activate members of the mitogen-activated protein kinase (MAPK) signaling:

- p38 kinase;

- extracellular signal-regulated kinase (ERK);

- c-jun N-terminal kinase (JNK);

- phosphoinositol-3 kinase.

All of the above have the potential to influence inflammation in asthmatic airways [7].

When compared to healthy subjects it is apparent that superoxide dismutase (SOD) activity is significantly lower in the epithelial lining fluid and airway epithelial cells of asthmatic subjects. Indeed, when segmental antigen was instilled in the lung of an asthmatic suffering from atopic asthma, it was clear within a few minutes that loss of SOD activity had occurred [3]. This loss was due to the twofold increase in $\mathrm{O} 2 \bullet^{-}$generation after antigen instillation into the airways of these atopic individuals. A link between the SOD activity levels and the level of airway reactivity has also been described. Earlier studies carried out in larger populations reported that airway reactivity is inversely related to SOD activity [3]. These findings support the notion that there is a link between SOD activity and physiologic parameters of asthma severity. Other studies have also indicated 
that the lower SOD activity in asthma occurred as a result of the increased oxidative and nitrosative stress in the asthmatic airways, which therefore can be used as a sensitive marker indicating the airway redox activity and asthma severity. When acute asthma attacks are taking place, there is an increase in the reactive oxygen and nitrogen species [3]. It can therefore be inferred that the loss of SOD also contributes to oxidative stress during acute asthma exacerbations. Reports have, in fact, shown that manganese (Mn)SOD is a target for tyrosine nitration and oxidation, which in turn results in a loss of enzyme function, and tissue injury. Considering data in relation to MnSOD oxidation and nitration in human asthmatic lungs, it can be seen that up to $10 \%$ of MnSOD recovered from asthmatic airway epithelial cells has at least one oxidative modification. It is uncertain, however, whether the average amount of modification of MnSOD can affect redox and cell functions in vivo, as oxidative modification/inhibition of MnSOD triggers apoptosis in airway epithelial cells in vitro. Apoptosis and shedding of epithelial cells has also been monitored in asthmatic patients [8]. This led to the finding that redox modifications of SOD may play a part in airway remodeling and epithelial apoptosis, which results in the shedding of the airway epithelial cells and predisposes to greater airway hyperreactivity. Recent studies have also indicated a loss of circulating SOD activity in asthmatics. Serum SOD activity is related to asthma lung function, and this finding appears to be unique to asthma. In vitro studies have shown that reactive oxygen and nitrogen species lead to oxidative and nitrative modifications of tyrosine and inactivation of $\mathrm{Mn}$ SOD, while copper- and zinc-containing superoxide dismutase (Cu/Zn SOD) can be inactivated by ROS and RNS through targeting of critical histidine residues and formation of histidinyl radicals.

In the airway epithelial cells of asthma subjects, the oxidative modification and inactivation of MnSOD is clearly apparent [9]. The increase in oxidative and nitrosative stress in these subjects is a result of the increased loss of SOD activity, which indicates that SOD may operate as a surrogate marker indicating the level of oxidant stress and asthma severity. Inhaled glucocorticosteroids are a staple of the therapy used for the majority of adults suffering with asthma. In addition cromolyn sodium, nedocromil, and leukotriene modifiers may also be used. In the event that inhaled drugs are not sufficient to relieve the asthmatic symptoms, oral corticosteroids are employed.

Is antioxidant therapy an alternative approach for treating asthma? Antioxidants have been shown to be effective in controlling ischemia/reperfusion injury, shock and lung injury which has occurred as a result of radio- and chemo-therapy, and in chronic inflammatory disorders such as rheumatoid arthritis and osteoarthritis [5]. Due to the fact that asthma is also associated with a decrease in antioxidant SOD, strategies aimed at increasing airway SOD levels may be a logical approach that could be adopted to ensure more effective therapy for asthmatics. Additional support for SOD therapy comes from murine models of asthma, in which a link between antioxidants and airway hyperresponsiveness has been shown. Indeed, it has been demonstrated that transgenic mice who over-express SOD have, as a result, decreased allergen-induced physiologic changes in the airways in comparison to controls [5]. Also, treatment with SOD mimetics reduces the magnitude of ovalbumin-induced airway hyperresponsiveness to methacholine in murine models of asthma [5]. Furthermore, exogenous SOD provided intratracheally to mice treated with asbestos has decreased the neutrophil influx and oxidative matrix degradation [5]. One study in animal models has suggested that therapies targeting redox-regulated signal transduction pathways are useful and can reduce airway inflammation [10].

However, manipulation of endogenous SOD for therapeutic purposes has been problematic due to its short half-life and large molecular weight. A number of SOD mimetics based on organ-manganese complexes have been developed that retain their antioxidant properties in vivo. One study highlighted the fact that SOD mimics given before antigen challenge of sensitised guinea pigs attenuate allergen-induced asthmatic bronchospasm [5]. All of these animal studies support the notion that SOD mimetics may be effective in treating asthmatic airway inflammation. Clinical trials on the effects of SOD mimetics in patients with asthma have not yet been performed. Hence, the aforementioned studies, although providing grounds for optimism, should nevertheless be viewed with due caution.

In this context, the study by Katsoulis and coauthors, included in this issue [11], showing decreased levels of erythrocyte SOD activity in patients suffering from an asthma attack and a significant correlation of erythrocyte SOD activity with functional parameters of patients at discharge, suggests a potential role for these measurements in monitoring the level of oxidative stress during asthma attacks and offers, as suggested by the authors, a potential approach for monitoring the health status and the pharmacological response of asthmatic patients.

However, two major questions were not addressed by the authors and remain open: 1) the analysis of SOD activity needs to be carried out over a longer period of time (1-3 months after the exacerbation event) in order to establish the enzymatic changes occurring in asthmatic airways and the potential recovery process occurring after the acute event; 2) the potential therapeutic use of antioxidants, in particular of SOD mimetics, during and after an asthma attack needs to be verified through specific studies designed to identify the most appropriate time and dosage course for treating asthmatic patients with these new pharmacologic agents. We believe that in the next few years a more detailed picture about the potential of these pharmacologic agents will emerge from more advanced research. 


\section{References}

1. Busse W, Elias J, Sheppard D, Banks-Schlegel S. Airway remodeling and repair. Am J Respir Crit Care Med 1999; 160: 1035-1042.

2. Hoshino T, Okamoto M, Takei S, Sakazaki Y, Iwanaga T, Aizawa H. Redox-regulated mechanisms in asthma. Antioxid Redox Signal 2008; 10: 769-783.

3. Comhair SA, Bhathena PR, Dweik RA, Kavuru M, Erzurum SC. Rapid loss of superoxide dismutase activity during antigen-induced asthmatic response. Lancet 2000; 355: 624 .

4. Sanders SP, Zweier JL, Harrison SJ, Trush MA, Rembish SJ, Liu MC. Spontaneous oxygen radical production at sites of antigen challenge in allergic subjects. Am J Respir Crit Care Med 1995; 151: 1725-1733.

5. Comhair SA, Erzurum SC. Redox control of asthma: molecular mechanisms and therapeutic opportunities. Antioxid Redox Signal 2010; 12: 321-52.

6. Montuschi P, Corradi M, Ciabattoni G, Nightingale J, Kharitonov SA, Barnes PJ. Increased 8-isoprostane, a marker of oxidative stress, in exhaled condensate of asthma patients. Am J Respir Crit Care Med 1999; 160: 216-220.

7. Carvalho H, Evelson P, Sigaud S, Gonzalez-Flecha B. Mitogen-activated protein kinases modulate $\mathrm{H}(2) \mathrm{O}(2)$ induced apoptosis in primary rat alveolar epithelial cells. J Cell Biochem 2004; 92: 502-513.

8. Bucchieri F, Puddicombe SM, Lordan JL, et al. Asthmatic bronchial epithelium is more susceptible to oxidant-induced apoptosis. Am J Respir Cell Mol Biol 2002; 27: 179-185.

9. Comhair SA, Xu W, Ghosh S, Thunnissen FB, et al. Superoxide dismutase inactivation in pathophysiology of asthmatic airway remodeling and reactivity. $A m J$ Pathol 2005; 166: 663-674.

10. Henderson WR, Jr, Chi EY, Teo JL, Nguyen C, Kahn M. A small molecule inhibitor of redox-regulated NFkappa B and activator protein-1 transcription blocks allergic airway inflammation in a mouse asthma model. $J$ Immunol 2002; 169: 5294-5299.

11. Katsoulis K, Kontakiotos T, Gerou S, Kougioulis M, Lithoxopoulou H, Papakosta D. Alterations of erythrocyte superoxide dismutase activity in patients suffering from asthma attacks. Monaldi Arch Chest Dis 2010; 73: 99-104.

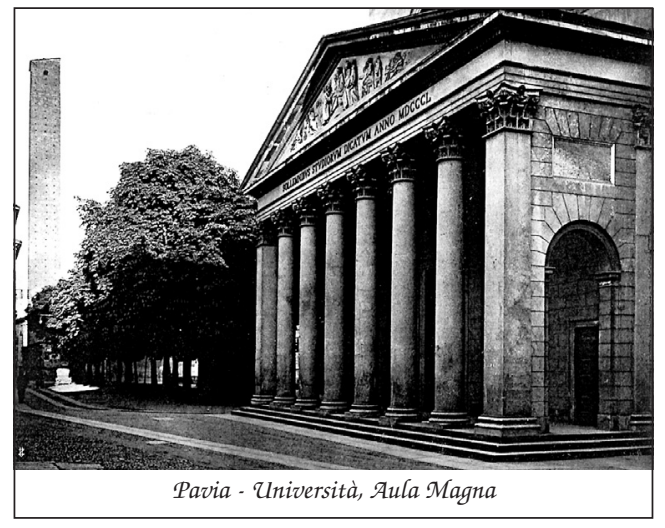

